\title{
NÚMEROS CROMOSSÔMICOS DE ESPÉCIES DE COMMELINACEAE R. BR. OCORRENTES NO NORDESTE DO BRASIL
}

\author{
SILVIA ROMEU PITREZ*, LEONARDO PESSOA FELIX**, ROXANA BARRETO* \\ \& MARCELO GUERRA* \\ * Departamento de Botânica, CCB, Universidade Federal de Pernambuco, R. Prof. Nelson Chaves, s.n., 50670-420 - Recife, \\ PE, Brasil \\ ** Departamento de Fitotecnia, CCA, Universidade Federal da Paraíba, Campus III, 58397-000 - Areia, PB, Brasil.
}

\begin{abstract}
Chromosome numbers of Commelinaceac R. Br. species occurring in the Northeast of Brazil). In this work, 46 populations belonging to 17 species and 8 gencra of Commelinaceac occurring in the Northeast of Brazil were cytologically analysed. Five of these specics (Aneilema brasiliense $2 \mathrm{n}=40$, Commelina rufipes var. rufipes $2 \mathrm{n}=30$, Dichorisandra albo-marginata $2 \mathrm{n}=76$, D. puberula $2 \mathrm{n}=38$ and Tinantia sprucei $2 \mathrm{n}=34$ ) had no previous chromosome number register in the literature. The chromosome numbers of the remaining species (Callisia filiformis $2 \mathrm{n}=14$, C. monandra $2 \mathrm{n}=14$, C. repens $2 \mathrm{n}=12$, Commelina benghalensis $2 \mathrm{n}=22$, C. diffusa $2 \mathrm{n}=30$, C. erecta $2 \mathrm{n}=60, C$. obliqua $2 \mathrm{n}=60$, Dichorisandra hexandra $2 \mathrm{n}=38, D$. thyrsiflora $2 \mathrm{n}=38$, Gibasis geniculata $2 \mathrm{n}=32$, Tradescantia ambigua $2 \mathrm{n}=24$, and Tripogandra glandulosa $2 n=16$ ) were in agreement with at least one of the previous reports. The interphase nuclei were semi-reticulate in ten species and reticulate in other scven. Meiotic pairing was normal in six of the analysed species (Aneilema brasiliense, Callisia filiformis, Dichorisandra puberula, Gibasis geniculata, Tradescantia ambigua and Tripogandra glandulosa), with exclusive formation of bivalents. However, a bridge was observed in most anaphase I cells of Tradescantia ambigua. Interpopulational variability was not observed. In the genus Dichorisandra chromosome size and morphology were relatively constant. Three specics showed $2 n=38$ whereas $D$. albo-marginata presented $2 n=76$. The wide range of chromosome numbers variation found in our sample confirms the known karyological diversity of Commelinaceac from other areas.
\end{abstract}

\begin{abstract}
Resumo - (Números cromossômicos de espécics de Commelinaceac R. Br. ocorrentes no Nordeste do Brasil). Nestc trabalho foram analisados citologicamente 46 populações pertencentes a 17 espécies de oito gêneros da família Commelinaceae ocorrentes no Nordeste Brasileiro. Cinco dessas cspécies (Aneilema brasiliense $2 \mathrm{n}=40$, Commelina rufipes var. rufipes $2 \mathrm{n}=30$, Dichorisandra albo-marginata $2 \mathrm{n}=76, D$. puberula $2 \mathrm{n}=38$, Tinantia sprucei $2 \mathrm{n}=34$ ) não apresentavam registro citogenético anterior na literatura. Nas demais espécies (Callisia filiformis $2 \mathrm{n}=14, C$. monandra $2 n=14, C$. repens $2 n=12$, Commelina benghalensis $2 n=22$, C. diffusa $2 n=30$, C. erecta $2 n=60$ c C. obliqua $2 n=60$, Dichorisandra hexandra $2 \mathrm{n}=38$, D. thyrsiflora $2 \mathrm{n}=38$, Gibasis geniculata $2 \mathrm{n}=32$, Tradescantia ambigua $2 \mathrm{n}=24$, Tripogandra glandulosa $2 \mathrm{n}=16$ ) os númcros cromossômicos obscrvados coincidiram com ao menos um dos registros prévios para cada espécie. Os núclcos interfásicos foram do tipo semi-reticulado $\mathrm{cm} 10$ espécics e reticulado nas outras scte. O pareamento meiótico de seis espécies analisadas (Aneilema brasiliense, Callisia filiformis, Dichorisandra puberula, Gibasis geniculata, Tradescantia ambigua c Tripogandra glandulosa) foi regular, com formação exclusiva de bivalentes. Contudo, foi observada uma pontc na maioria das células $\mathrm{cm}$ anáfasc I de Tradescantia ambigua. Variações interpopulacionais não foram observadas. No gênero Dichorisandra o tamanho c a morfologia cromossômica foram relativamente constantes. Três espécies apresentaram $2 \mathrm{n}=38$ c $D$. albomarginata apresentou $2 n=76$. Uma ampla variação numérica foi encontrada entre as cspécics analisadas, confirmando a diversidade cromossômica observada cm comelináccas de outras regiões.
\end{abstract}

Key words: chromosome numbers, Commelinaceac, interphase nuclei.

\section{Introdução}

A família Commelinaceae tem ampla distribuição nos trópicos e subtrópicos de todo o mundo, com gêneros comuns às regiões paleotropicais e neotropicais (Faden \& Hunt 1991). Possui cerca de 620 espécies (Mabberley 1987) pertencentes a 38 gêneros (Faden \& Hunt 1991) dos quais 13 gêneros e cerca de 61 espécies são referidos para o Brasil, onde nove gêneros e 33 espécies são citadas para o Nordeste brasileiro (Barreto 1997).

Devido principalmente ao maior tamanho de seus cromossomos e à ocorrência freqüente de alterações cromossômicas numéricas e estruturais, vários trabalhos citotaxonômicos foram desenvolvidos com as comelináceas, utilizando técnicas de coloração convencional (ver, por exemplo, Jones \& Jopling 1972), bandeamento C (Kenton 1978), coloração com fluorocromos (Kenton 1991) e hibridização in situ (Parokonny et al. 1992).

Apesar do uso cada vez maior de técnicas citogenéticas mais refinadas, o principal parâmetro citotaxonômico continua sendo a variabilidade cromossômica numérica (Guerra 2000).
A análise da variação cromossômica numérica de um taxon permite reconhecer o número básico do grupo, o qual constitui o principal instrumento para análise citotaxonômica. Nesse sentido, o presente trabalho objetiva contribuir para o conhecimento citológico das espécies de Commelinaceae ocorrentes no Nordeste brasileiro. As técnicas convencionais de coloração permitem também observar o tamanho, a morfologia cromossômica, o número e posição dos satélites e a estrutura da cromatina nos núcleos interfásicos. Todos esses parâmetros são importantes para entender as mudanças ocorridas nos números básicos e a evolução cariológica do grupo, particularmente na família Commelinaceae (ver Jones \& Jopling 1972)

Apesar de ser uma família bastante explorada citogeneticamente, apenas 13 das 61 espécies ocorrentes no Brasil foram anteriormente estudadas (Jones \& Jopling 1972, Dias 1980, Guerra 1986, Boaventura \& Matthes 1987, Beltrão \& Guerra 1990, Carvalheira et al. 1991, Cruz et al. 1993). Neste trabalho são apresentados dados citogenéticos de 46 amostras pertencentes a 17 espécies de oito gêneros de Commelinaceae, 
ocorrentes na região Nordeste do Brasil. Esses resultados são comparados e analisados em relação aos dados para essas espécies noutras regiões.

\section{Material e Métodos}

Todo material foi coletado na região nordeste do Brasil, nos estados de Pernambuco, Paraíba e Piauí, com exsicatas depositadas nos herbários UFP, da Universidade Federal de Pernambuco, e HST, da Universidade Federal Rural de Pernambuco (abreviaturas de acordo com Mori et al. 1989). A tabela 1 traz a relação das espécies analisadas, suas respectivas referências de herbário e locais de coleta.

Foram analisados os cromossomos mitóticos de todas as espécies coletadas, bem como o comportamento meiótico de seis delas: Aneilema brasiliense, Callisia filiformis, Dichorisandra puberula, Gibasis geniculata, Tradescantia ambigua e Tripogandra glandulosa.

Para análise mitótica, pontas de raízes foram coletadas e pré-tratadas com 8 -hidroxiquinoleína $2 \mathrm{mM}$ por 20 a 24 horas a $10^{\circ} \mathrm{C}$. Posteriormente, foram fixadas em Carnoy (álcool etílico/ácido acético, $3: 1, \mathrm{v} / \mathrm{v}$ ), por 5 a 24 horas à temperatura ambiente e estocadas em "freezer" por tempo indeterminado. Para a preparação das lâminas, as pontas de raízes foram retiradas do fixador e lavadas duas vezes em água destilada por cinco minutos. Em seguida, hidrolizadas em $\mathrm{HCl} 5 \mathrm{~N}$ por 20 minutos à temperatura ambiente, lavadas em água destilada e o meristema esmagado em ácido acético a $45 \%$. As lamínulas foram retiradas por congelamento em nitrogênio líquido e as lâminas postas para secar à temperatura ambiente. Posteriormente, foram coradas convencionalmente com Giemsa a $2 \%$ (Guerra 1983) ou hematoxilina a 1\% (Guerra 1999). Para meiose, anteras jovens foram fixadas diretamente em Carnoy. O preparo das lâminas seguiu a mesma metodologia da análise mitótica, reduzindo-se porém o tempo de hidrólise para cinco minutos.

As fotografias foram feitas em fotomicroscópio Leica DMRB, utilizando filme Imagelink HQ 25 ASA da Kodak e copiadas em papel Kodabromide F3, Kodak.

\section{Resultados e Discussão}

No presente trabalho foram analisadas a morfologia, a variabilidade cromossômica numérica, bem como o número e a posição dos satélites e o tipo de núcleo interfásico em 18 espécies da família Commelinaceae. Variações interpopulacionais não foram detectadas em nenhuma das espécies analisadas para nenhum dos parâmetros observados.

Com relação à estrutura dos núcleos interfásicos, as quatro espécies de Dichorisandra, Tradescantia ambigua, Callisia repens e Tripogandra glandulosa apresentaram núcleos reticulados. As 10 espécies restantes apresentaram núcleos interfásicos do tipo semi-reticulado, com retículo fracamente corado e cromocentros de formato irregular.
A tabela 1 apresenta a relação dos números cromossômicos observados e o registro prévio encontrado para 12 das 17 espécies estudadas. Para Tinantia sprucei, Dichorisandra albo-marginata, D. puberula, Aneilema brasiliense e Commelina rufipes var. rufipes, não foi encontrado nenhum registro citogenético anterior.

Dichorisandra é o gênero de maior representação no Brasil, onde ocorrem 23 espécies (Barreto 1997). As espécies $D$. hexandra, D. puberula e D. thyrsiflora apresentaram $2 \mathrm{n}=38$ (Figuras $1 \mathrm{~A}-\mathrm{C}$ ), enquanto D. albo-marginata $(2 \mathrm{n}=76$ ) foi tetraplóide em relação às demais espécies (Figura 1D). Esse é o segundo registro de poliploidia em Dichorisandra e em toda a subtribo Dichorisandrinae, onde o número cromossômico $2 \mathrm{n}=38$ é dominante (Anderson \& Sax 1936, Jones \& Jopling 1972, Faden \& Hunt 1991). Em uma espécie não identificada, procedente do Peru, Jones \& Jopling (1972) observaram $2 \mathrm{n}=76$ mais dois cromossomos B. A morfologia cromossômica foi relativamente constante no gênero, com cromossomos grandes, submetacêntricos a acrocêntricos. Em algumas células foram observados satélites nos braços curtos de um dos pares acrocêntricos de $D$. hexandra, $D$. puberula e $D$. thyrsiflora e em dois pares acrocêntricos de $D$. albomarginata. A meiose de $D$. puberula apresentou-se regular, com 19 bivalentes. A localização dos quiasmas foi sempre em ambos os terminais cromossômicos, dando aos bivalentes uma morfologia característica de anel, exceto em um ou dois bivalentes que apresentaram apenas um quiasma, resultando numa forma de bastão (Figura 1B).

Em Tinantia sprucei foi observado $2 \mathrm{n}=34$, destacandose um par satelitado entre os cromossomos menores do complemento (Figura 1E). A única espécie de Tinantia com número cromossômico previamente conhecido era $T$. erecta (Jacq.) Schlecht. Jones \& Jopling (1972) descreveram uma ampla variação de números cromossômicos entre indivíduos procedentes da Guatemala, Peru e de quatro diferentes localidades da Argentina ( $2 n=34,34+2 f, 66-68)$ e em um exemplar do jardim botânico de Copenhague $(2 \mathrm{n}=c a 128)$. Os dois fragmentos relatados por esses autores podem corresponder aos satélites observados em $T$. sprucei, freqüentemente encontrados muito afastados dos cromossomos. A grande similaridade entre o cariótipo com $2 n=34$ apresentado por esses autores e o do presente material sugere que $x=17$ seja $o$ número básico do gênero.

Gibasis geniculata é a única espécie desse gênero que ocorre no Brasil (Barreto 1997) e uma das duas que ocorrem na América do Sul, formando a seç̧ão Heterobasis (Jones et al. 1975). Os números cromossômicos encontrados neste estudo foram $n=16$ (Figura 2A), com pareamento e segregação de homólogos normal, e $2 n=32$. Jones et al. (1975) encontraram $2 \mathrm{n}=32 \mathrm{em}$ três amostras dessa espécie procedentes de Belize e uma do México. Esses mesmos autores reportaram ainda $2 n=48$ em uma população da Argentina e outra de Trindade, confirmando o registro de Simmonds (1954). Contagens anteriores de $2 n=16$ para essa espécie (Tabela 1) são provavelmente devidas a dificuldades na identificação taxonômica (ver 
Jones et al. 1975). Ao contrário das espécies sul-americanas, os representantes de Gibasis na América Central apresentam números cromossômicos muito variados, cromossomos grandes, fusões/fissões Robertsonianas, complexos de translocação, cromossomos B, etc. (ver, por exemplo, Handlos 1970, Kenton 1991, Parokonny et al. 1992).

O gênero Tradescantia está representado no Brasil por oito espécies, porém, apenas T. ambigua, é encontrada na região Nordeste (Barreto 1997). De todas as espécies aqui estudadas esta foi a que apresentou cariótipo mais simétrico, com 24 cromossomos metacêntricos (Figura 2B). Satélites não foram observados. Este mesmo número foi reportado por Jones \& Jopling (1972), Jones \& Kenton (1984) e Martinez \& Ginzo (1985) para T. ambigua da Argentina. A análise meiótica revelou a frequente ocorrência de uma ponte em anáfase I, algumas vezes acompanhada de um pequeno fragmento cromossômico aparentemente acêntrico. Esse mesmo tipo de alteração meiótica tem sido também observado em outras espécies do gênero e sua ocorrência é provavelmente devida à inversões paracêntricas em heterozigose (Swanson, 1940).

As espécies do gênero Callisia apresentaram-se divididas em dois grupos, de acordo com as características cariotípicas. Em um deles se encontram $C$. filiformis e $C$. monandra, ambas com $2 \mathrm{n}=14$, núcleos interfásicos do tipo semi-reticulado e cromossomos profásicos apresentando condensação tardia na região terminal. No outro, está $C$. repens $\operatorname{com} 2 \mathrm{n}=12$, núcleo interfásico reticulado e cromossomos profásicos com condensação uniforme. Variações bruscas na estrutura do núcleo interfásico, entre espécies de um mesmo gênero, são relativamente raras e geralmente indicam a existência de subgêneros distintos dentro do gênero. Em Passiflora, por exemplo, essa mudança está associada aos subgêneros que apresentam diferentes números básicos (Melo et al., no prelo). Uma análise detalhada do cariótipo dessas espécies e suas implicações citotaxonômicas serão discutidas em outro trabalho (Pitrez \& Guerra, em preparação).

Barreto (1997) citou para o Brasil a ocorrência de cinco espécies do gênero Tripogandra, sendo este o primeiro registro de T. glandulosa para o Nordeste. O cariótipo apresentou um par metacêntrico grande e sete acrocêntricos, sendo dois pares maiores e cinco menores, todos sem satélites visíveis (Figura 2C). O comportamento meiótico foi normal com formação de 8 bivalentes, destacando-se um par cromossômico com segregação precoce em metáfase I. Apesar de haver uma grande variação cromossômica numérica no gênero, o número básico mais provável para o gênero é $x=8$ e $o$ cariótipo do presente material coincide com o descrito em exemplares da Argentina e Honduras (Handlos 1970, Jones \& Jopling 1972).

Aneilema é um gênero pantropical, com cerca de 62 espécies, das quais apenas duas ocorrem no Brasil (Barreto 1997). Em A. brasiliense, o número cromossômico somático foi $2 \mathrm{n}=40$ (Figura 2D) e a meiose mostrou comportamento regular com 20 bivalentes. Satélites não foram observados nas células analisadas. $O$ gênero possui ampla variação numérica e necessita de uma revisão taxonômica extensa (Jones \& Jopling 1972). A única outra espécie ocorrente no Brasil (A. umbrosum Kunth subsp. ovato-oblongum P. Beauv.) apresenta $2 n=26$, um número cromossômico fortemente divergente (Moore 1973).

O gênero Commelina apresenta cerca de 170 espécies e distribuição cosmopolita (Faden \& Hunt 1991), sendo que apenas oito espécies foram registradas para o Brasil (Barreto 1997). Cinco espécies (C. benghalensis, $C$. diffusa, C. erecta, C. obliqua e $C$. rufipes var. rufipes) foram analisadas neste trabalho, todas com cromossomos pequenos a médios e núcleos interfásicos semi-reticulados, características que parecem ser comuns ao gênero. Entre essas, destaca-se $C$. benghalensis, que apresentou sempre $2 \mathrm{n}=22 \mathrm{em}$ cinco populações analisadas. O cariótipo mostrou nove pares submetacêntricos e dois pares acrocêntricos, com um satélite no braço curto de um dos acrocêntricos (Figura 2E). Essa espécie é citologicamente atípica por apresentar cromossomos maiores que as demais, além de ser uma das poucas com $n=11$, enquanto nas outras cerca de 70 espécies citologicamente conhecidas predomina $\mathrm{x}=15$ (Lewis 1964). O número $2 \mathrm{n}=22$ havia sido reportado anteriormente para diversas populações de $C$. benghalensis da África e Índia (ver, por exemplo, Lewis 1964, Alam \& Sharma 1991). C. diffusa (Figura 2F) e C. rufipes var. rufipes (Figura 2G) mostraram um complemento cromossômico com $2 n=30$, com cromossomos acrocêntricos a metacêntricos, observadose na primeira um dos pares acrocêntricos satelitado. As outras duas espécies, $C$. obliqua e $C$. erecta foram muito semelhantes, em suas características morfológicas, diferindo basicamente pela ocorrência de folhas com base assimétrica na primeira (Barreto 1997). Cariologicamente, entretanto, apesar de possuírem o mesmo número cromossômico $(2 \mathrm{n}=60)$, C. erecta diferenciou-se de $C$. obliqua por apresentar cromossomos ligeiramente maiores, com dois pares satelitados (Figuras $2 \mathrm{H}$ J). Exceto para C. rufipes var. rufipes, que teve o primeiro registro cromossômico neste trabalho, os dados para as demais espécies confirmaram resultados de autores anteriores (Tabela 1). O fato de que em todas as quatro espécies previamente analisadas foram encontrados mais de um número cromossômico (Tabela 1) é, ao menos em parte, devido à dificuldade de identificação taxonômica e a contagens imprecisas (Jones \& Jopling 1972, Guerra 2000). Uma análise citotaxonômica mais detalhada, certamente permitirá uma caracterização mais segura das espécies desse gênero.

Os dados para as comelináceas brasileiras mostram que a família apresenta uma grande diversidade de número e morfologia cromossômica, embora nenhuma variação intraespecífica tenha sido encontrada nas diversas populações analisadas neste trabalho. Uma estabilidade semelhante foi observada em populações de várias outras espécies do nordeste brasileiro (ver, por exemplo, Beltrão \& Guerra 1990, Felix \& Guerra 1998, no prelo). Esses dados sugerem que a flora dessa região possa ter um padrão evolutivo mais estável que o reconhecido para outras regiões tropicais (Stebbins 1971, Guerra 1990). 

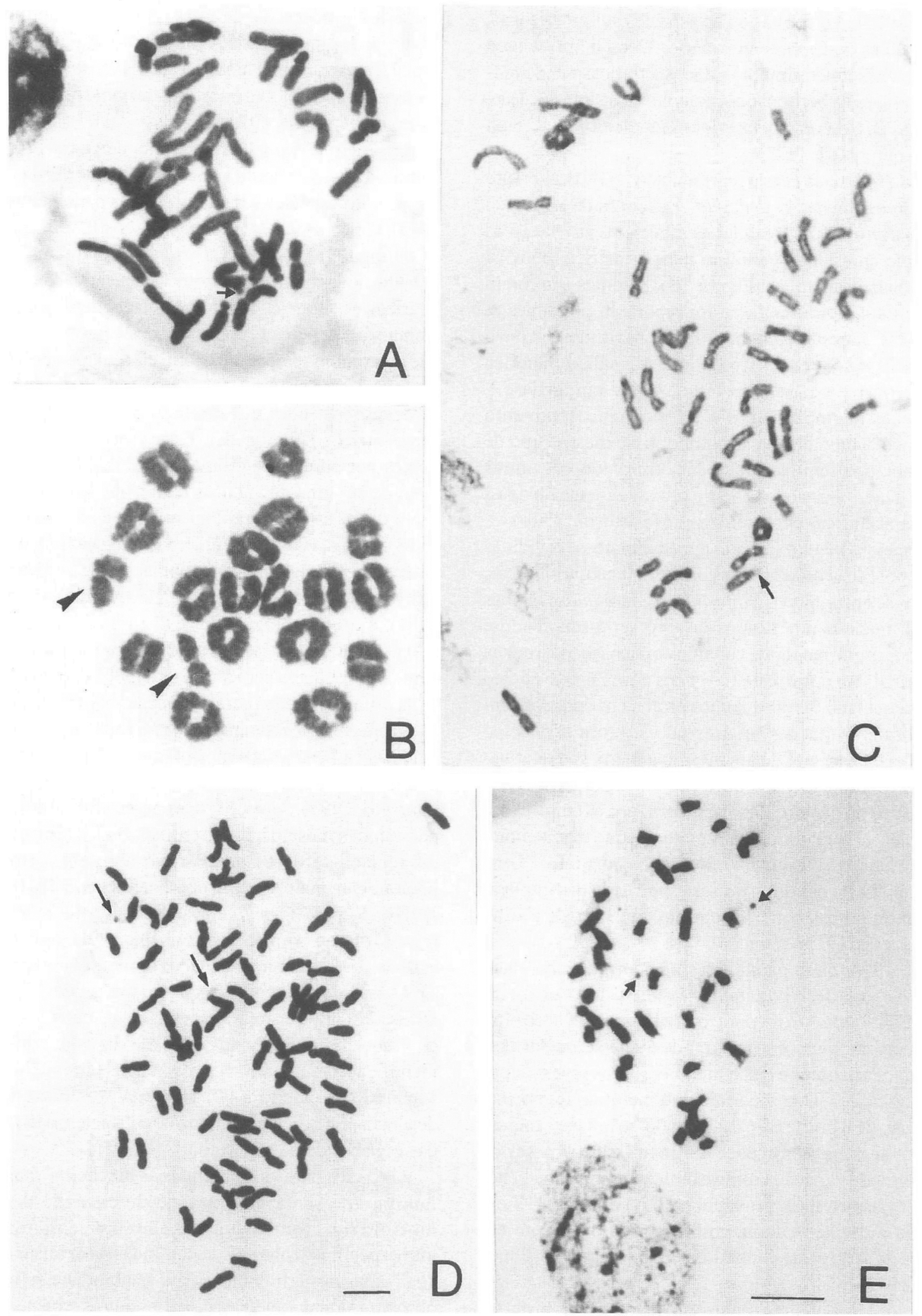

Fig. 1. Complementos cromossômicos de espécies de Dichorisandra e Tinantia. A- Metáfase mitótica de Dichorisandra hexandra com 2n=38. B- Diacinese de D. puberula com 19 bivalentes. Cabeças de setas indicam bivalentes com um único quiasma terminal. C- Metáfase mitótica de $D$. thyrsiflora $\operatorname{com} 2 \mathrm{n}=38$. D- Metáfase mitótica de $D$. albo-marginata com $2 \mathrm{n}=76$. E- Metáfase mitótica e núcleo interfásico de Tinantia sprucei com 2n=34. Setas nas figuras A, C, D e E apontam satélites. Fig. A, B, C e E, na mesma ampliação; D em ampliação menor. Barras nas figuras D e E correspondem a $10 \mu \mathrm{m}$. 


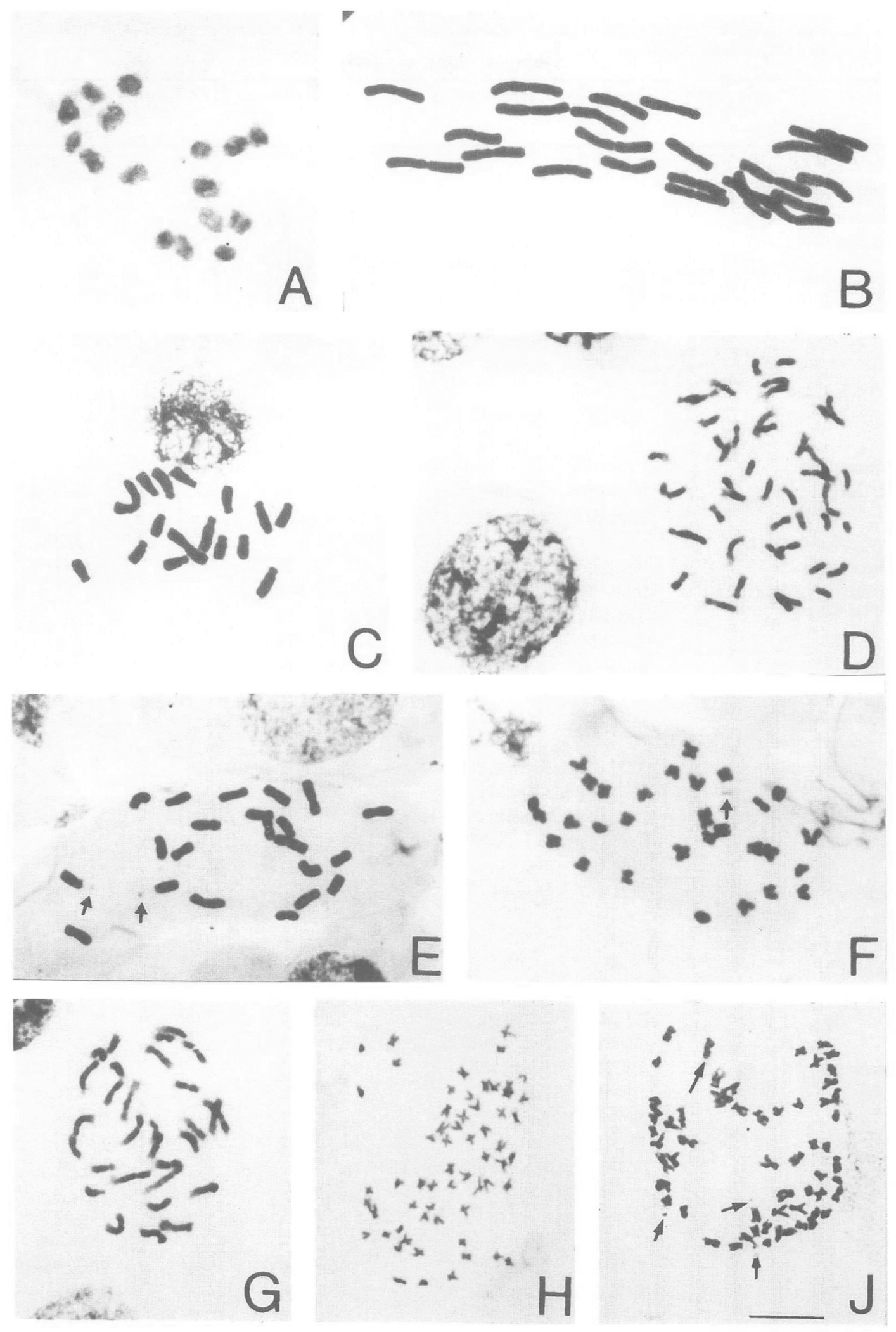

Fig. 2. Complementos cromossômicos de espécies de Gibasis, Tradescantia, Tripogandra, Aneilema e Commelina. A- Diacinese de Gibasis geniculata com 16 bivalentes. B- Metáfase mitótica de Tradescantia ambigua com $2 \mathrm{n}=24$. C- Metáfase de Tripogandra glandulosa com 2n=16. D- Prometáfase e núcleo interfásico de A. brasiliense $(2 \mathrm{n}=40)$. E- Metáfase de $C$. benghalensis com $2 \mathrm{n}=22$. F- Metáfase de $C$. diffusa $\operatorname{com} 2 \mathrm{n}=30$. G- Metáfase de $C$. rufipes var. rufipes $\operatorname{com} 2 \mathrm{n}=30$. H- Metáfase de $C$. obliqua com $2 \mathrm{n}=60$. J-Metáfase de $C$. erecta com $2 \mathrm{n}=60$. Satélites são indicados por setas. Barra em $\mathbf{J}$ corresponde a $10 \mu \mathrm{m}$. 
Tabela 1. Lista dos taxa analisados, organizados segundo Faden e Hunt (1991), com as respectivas referências de herbário, localidades, números cromossômicos observados e contagens prévias.

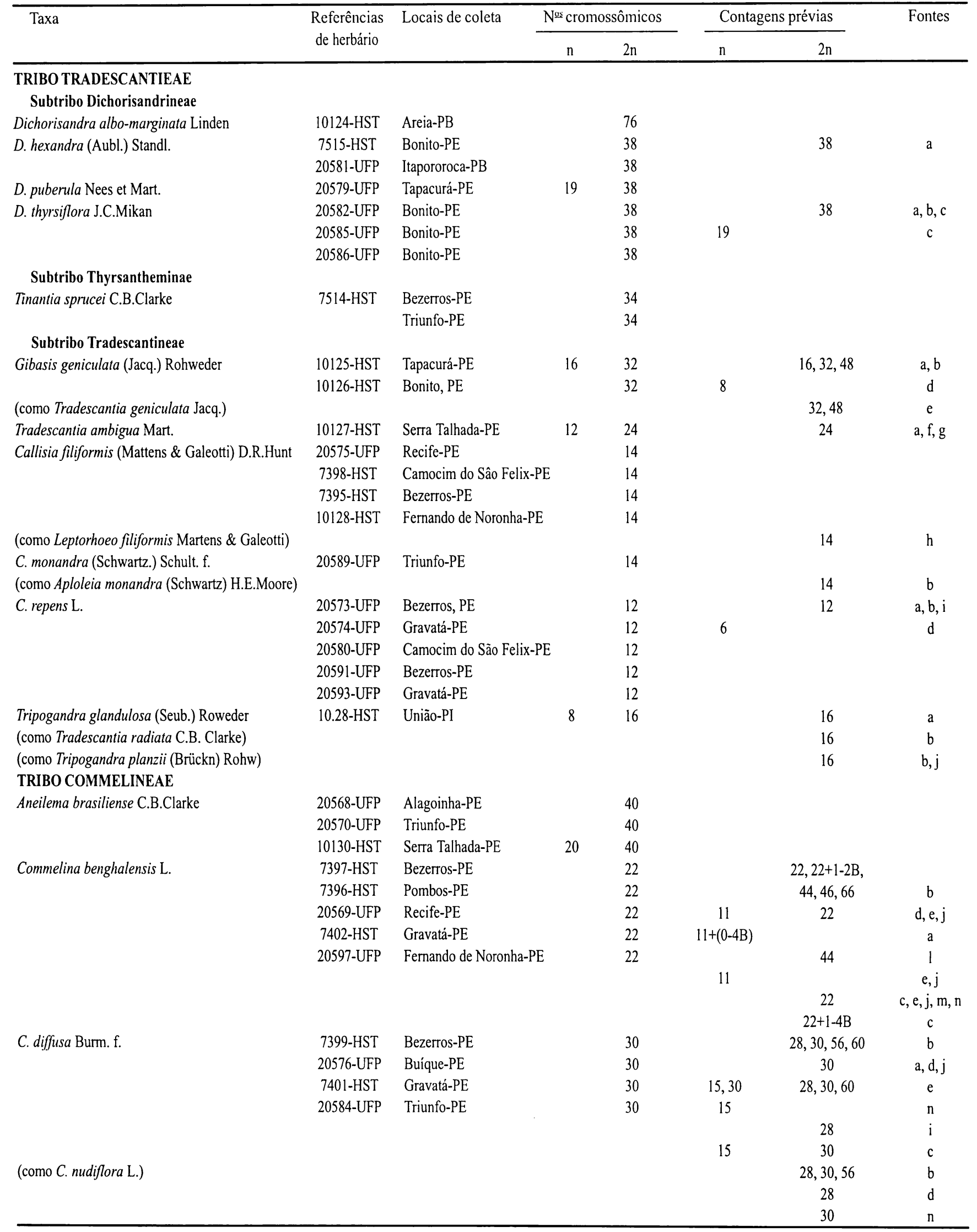


Tabela 1. Continuação.

\begin{tabular}{|c|c|c|c|c|c|c|c|}
\hline \multirow[t]{2}{*}{ Taxa } & \multirow{2}{*}{$\begin{array}{l}\text { Referências } \\
\text { de herbário }\end{array}$} & \multirow[t]{2}{*}{ Locais de coleta } & \multicolumn{2}{|c|}{ №s cromossômicos } & \multicolumn{2}{|c|}{ Contagens prévias } & \multirow[t]{2}{*}{ Fontes } \\
\hline & & & $\mathrm{n}$ & $2 n$ & $\mathrm{n}$ & $2 n$ & \\
\hline \multirow[t]{2}{*}{ C. erecta $\mathrm{L}$. } & 10131-HST & Recife-PE & & 60 & & $56,60, \approx 120$ & $b$ \\
\hline & 20596-UFP & Camaragibe-PE & & 60 & 30,60 & & $\mathrm{j}$ \\
\hline \multirow[t]{8}{*}{ C. obliqua Vahl } & 7400-HST & Bezerros-PE & & 60 & & $45,60,120,150$ & $\mathrm{~b}$ \\
\hline & 20587-UFP & Bezerros-PE & & 60 & 30 & & $d$ \\
\hline & 20571-UFP & Bezerros-PE & & 60 & & ca. 60 & a \\
\hline & 20590-UFP & Bonito-PE & & 60 & 60 & & $\mathrm{e}$ \\
\hline & 20583-UFP & Camaragibe-PE & & 60 & & & \\
\hline & 20595-UFP & Camaragibe-PE & & 60 & & & \\
\hline & 20572-UFP & Itapororoca-PB & & 60 & & & \\
\hline & 20598-UFP & Fernando de Noronha-PE & & 60 & & & \\
\hline C. rufipes Seub. var. rufipes Seub. & 10132-HST & Bonito-PE & & 30 & & & \\
\hline
\end{tabular}

a, Moore 1974; b, Fedorov 1969; c, Goldblatt \& Johnson 1996; d, Moore 1973; e, Goldblatt 1981; f, Jones \& Kenton 1984; g, Martinez \& Ginzo 1985; h, Carvalheira et al. 1991; i, Goldblatt \& Johnson 1994; j, Goldblatt 1984; i, Moore 1977; m, Goldblatt 1988; n, Goldblatt \& Johnson 1990.

\section{Agradecimentos}

Os autores agradecem aos colegas Ângela Maria de Miranda Freitas, Reginaldo de Carvalho, George Baracho e José Alves, pela coleta de vários exemplares analisados no presente trabalho. A execução deste projeto foi financiada pela CAPES, FACEPE e CNPq.

\section{Referências}

ALAM, N. \& SHARMA, A.K. 1991. In situ absorbance and chromosome characteristics in Commelinaceac. In R. C. Sobti, G. Obc (cds) Eukaryotic chromosomes: strutural and functional aspects. SpringerVerlag, New York, p. 47-51.

ANDERSON, E. \& SAX, K. 1936. A cytological monograph of the Amcrican species of Tradescantia. Bot. Gaz. 97: 433-475.

BARRETO, R.C. 1997. Levantamento das espécies de Commelinaceae R. Br. nativas do Brasil. Tese de Doutorado. Universidade de São Paulo. São Paulo.

BELTRÃO, G.T.A. \& GUERRA, M. 1990. Citogenćtica de angiospermas colctadas em Pernambuco - III. Ci. \& Cult. 42: 839-845.

BOAVENTURA, Y.M.S. \& MATTHES, L.A.F. 1987. Aspectos da biologia da reprodução $\mathrm{cm}$ plantas ornamentais cultivadas no Estado de São Paulo. I - Dichorisandra thyrsiflora Mikan (Commelinaccae). Acta Bot. Brasil. 1: 189-199.

CARVALHEIRA, G.M.G., GUERRA, M., SANTOS, G.A., ANDRADE, V.C. \& FARIAS, M.C.A. 1991. Citogenética de angiospermas coletadas cm Pernambuco - IV. Acta bot. bras. 5: 37-51.

CRUZ, N.D., BOAVENTURA, Y.M.S., CONAGIN, C.H.T.M., DUTILH, J.H.A., FORNI-MARTINS, E.R., MEDINA, D.M., MENDES, A.J.T., PIEROZZI, N.I. \& PINTO-MAGLIO, C.A.F. 1993. Citogenética vegetal: Cinqüienta e três anos de pesquisa da seção de citologia do Instituto Agronômico. Instituto Agronômico. Campinas.

DIAS, M.G.L. 1980. Efeito da temperatura no comportamento mciótico de Rhoeo spathacea (Swartz) Stearn (1788). Dissertação de Mestrado. Universidade Federal do Ceará. Fortaleza.

FADEN, R.B \& HUNT, D.R. 1991. The classification of the Commclinaceac. Taxon 40: 19-31.

FEDOROV, A.M.A. 1969. Chromosome numbers of flowering plants. Komarov Botanical Institute. Leningrado.

FELIX, L.P. \& GUERRA, M. 1998. Cytogenetic studies on species of Habenaria (Orchidaceae) occurring in the Northeast of Brazil. Lindleyana 13: 224-230.
FELIX, L.P. \& GUERRA, M. Cytogenetics and cytotaxonomy of some Brazilian specics of Cymbidioid orchids. Gen. Mol. Biol (no prelo).

GOLDBLATT, P. 1981. Index to plant chromosome numbers 1975-1978. Missouri Botanical Garden. St. Louis.

GOLDBLATT, P. 1984. Index to plant chromosome numbers 1979-1981. Missouri Botanical Garden. St. Louis.

GOLDBLATT, P. 1988. Index to plant chromosome numbers 1984-1985. Missouri Botanical Garden. St. Louis.

GOLDBLATT, P. \& JOHNSON, D.E. 1990. Index to plant chromosome numbesr 1986-1987. Missouri Botanical Garden. St. Louis.

GOLDBLATT, P. \& JOHNSON, D.E. 1994. Index to plant chromosome numbesr 1990-1991. Missouri Botanical Garden. St. Louis.

GOLDBLATT, P. \& JOHNSON, D.E 1996. Index to plant chromosome numbers 1992-1993. Missouri Botanical Garden. St. Louis.

GUERRA, M. 1983. O uso do Giemsa na citogenética vegetal - comparação entre a coloração simples e o bandeamento Ci. \& Cult. 35: 190-193.

GUERRA, M.S. 1986. Citogenćtica de angiospermas coletadas em Pcrnambuco. I. Revista Brasil. Genét. 9: 21-40.

GUERRA, M.S. 1990. A situação da citotaxonomia de angiospermas nos trópicos c, cm particular, no Brasil. Acta Bot. Brasil. 4: 75-86.

GUERRA, M. 1999. Haematoxylin: a simple, multiple-use dye for chromosome analysis. Gen. Mol. Biol. 22: 77-80.

GUERRA, M. 2000. Chromosome number variation and cvolution in monocots. In Wilson. K.L. \& Monison, D.A (eds) Monocots: Systematics and Evolution. CSIRO Publ., Melbourne, p. 127-136.

HANDLOS, W.L. 1970. Cytological investigations of some Commclinaceae from Mcxico. Baileya 17: 6-33.

JONES, K. \& JOPLING, C. 1972. Chromosomes and the classification of the Commelinaccac. Bot. J. Linn. Soc. 65: 129-162.

JONES, K. \& KENTON, A. 1984. Mechanisms of chromosome change in the cvolution of the tribe Tradescantieac (Commelinaceac). In Sharma, A.K. \& Sharma, A. (eds) Chromosomes in evolution of eukaryotic groups. CRC Press. Boca Raton, p. 143-168.

JONES, K., PAES, D. \& HUNT, D.R. 1975. Contributions to the cytotaxonomy of the Commelinaceac. II. Further observations on Gibasis geniculata and its allies. Bot. J. Linn. Soc. 71: 145-166.

KENTON, A. 1978. Gicmsa C-banding in Gibasis (Commelinaceac). Chromosoma 65: 309-324.

KENTON, A. 1991. Heterochromatin accumulation, disposition and diversity in Gibasis karwinskyana (Commelinaccac). Chromosoma 100: $467-478$.

LEWIS, W.H. 1964. Mciotic chromosomes in African Commelinaceae. Sida 1: $274-293$.

MABBERLEY, D.J. 1987. Pallas's Buckthorn and two and a half centuries of neglected binomials. Taxon 33: 433-444. 
MARTINEZ, A. \& GINZO, H. 1985. DNA content in Tradescantia. Can. J. Genet. Cytol. 27: 766-775.

MELO, N.F.; CERVI, A.C. \& GUERRA, M. Karyology and cytotaxonomy of the genus Passiflora L. (Passifloraccac). Pl. Syst. Evol. (no prelo).

MOORE, R.J. 1973. Index to plant chromosome numbers 1967-1971. Regnum Vegetabile 90: 1-539.

MOORE, R.J. 1974. Index to plant chromosome numbers for 1972. Regnum Vegetabile 91: 1-108.

MOORE, R.J. 1977. Index to plant chromosome numbers for 1973-1974. Regnum Vegetabile 96: 1-257.
MORI, S.A. SILVA, L.A.M., LISBOA, G. \& CORADIN, L. 1989. Manual de Manejo do Herbário Fanerogâmico. cd. 2. CEPLAC. Ilhćus.

PAROKONNY, A.S., KENTON, A.Y., MEREDITH, L., OWENS, S.J. \& BENNETT, M.D. 1992. Genomic divergence of allopatric sibling species studied by molecular cytogenetics of their $\mathrm{F}_{1}$ hybrids. Pl. Journal 2: 695-704.

SIMMONDS, N.W. 1954. Chromosome behaviour in some tropical plants. Heredity 8: 139-145.

STEBBINS, G.L. 1971. Chromosomal evolution in higher plants. AddisonWesley. London.

SWANSON,C.R. 1940. The distribution of inversions in Tradescantia. Genetics 25: 438-465. 